\title{
Les déterminants de la pluriactivité des ménages agricoles en Algérie
}

\author{
Radhia Bouchakour*, Slimane Bedrani ${ }^{* *}$, Omar Bouazouni ${ }^{\star * \star}$
}

DOI : $10.30682 / \mathrm{nm} 1802 \mathrm{~g}$

JEL codes: Q12, A14, C25

\begin{abstract}
Pluriactivity is a widespread phenomenon that has been credited for helping the survival as well as the demise of the rural farm. Given the prevalence of poverty in rural areas, farmers have always relied on non-farm income to survive. But the recent shrinking of rural areas has also raised the question of whether pluriactivity has helped the disappearance of farms. This paper explores four main determinants of pluriactivity and extends prior work by considering their relation to the neoclassical and portfolio theories. The context is that of a developing country as the existing literature has so far largely neglected this phenomenon within developing countries. We empirically test a set of four hypotheses drawn both from theory and prior empirical work.
\end{abstract}

Keywords: Pluriactivity, Off-farm work, Rural areas, Portfolio theory, Theory of labour supply.

\section{Introduction}

Il y a plusieurs décennies, la pluriactivité a était reconnue comme un phénomène mondial (Cavazzani et Fuller, 1982). Les agriculteurs comptent de plus en plus sur les revenus non agricoles, reflétant l'importance de la pluriactivité dans la survie de l'agriculture et de la paysannerie. Les enquêtes menées dans plusieurs pays montrent que jusqu'à deux tiers des agriculteurs ont déclaré avoir eu un certain nombre d'activités non agricoles (Kimhi, 2000).

Dans les pays en voie de développement, la pluriactivité est principalement un moyen de survie. L'agriculture ne garantit pas un revenu suffisant pour $72 \%$ des pauvres qui vivent dans les zones rurales (Banque mondiale, 1997). Le travail hors ferme a également été pratiqué depuis longtemps dans les pays industrialisés. Cependant, la préoccupation principale dans les pays développés est la disparition des exploitations. Par exemple, dans les zones rurales des États-Unis, le nombre de fermes a diminué continuellement depuis des décennies (Goetz et Debertin, 2001).

La grande majorité des études empiriques ont porté sur une poignée d'économies industrialisées. En revanche, la pluriactivité dans les pays en voie de développement a été largement négligée.

\footnotetext{
* Université Saad Dahleb, Blida, Algérie.

** Ecole National Supérieur Agronomique, Alger, Algérie.

*** Conseil National Economique et Social, Alger, Algérie.

Nous remercions Omar Bessaoud, IAMM/ CIHEAM, Montpellier, France, pour son aide précieuse.

Corresponding author: s.bedrani@ensa.dz.
} 
Notre contribution est de considérer la pluriactivité dans les exploitations d'un pays en voie de développement, à savoir l'Algérie. Bien que les pays en voie de développement soient hétérogènes, nous soutenons que nous pouvons quand même tirer quelques leçons de ces données étant donné que l'Algérie partage beaucoup de caractéristiques des pays en voie de développement. Par exemple, les agriculteurs algériens ont rencontré des problèmes semblables aux problèmes des agriculteurs d'autres pays en voie de développement, notamment la sécheresse, l'incertitude et la concurrence.

Dans cet article, la pluriactivité décrit une situation dans laquelle un agriculteur n'est pas entièrement investi (en termes de force de travail et/ou de capital) dans les activités agricoles. La pluriactivité est parfois appelée agriculture à temps partiel, ou travail hors ferme (Mishra et Goodwin, 1997).

L'article est divisé en 6 sections. La section 1 formant l'introduction, la section 2 donne un aperçu sur la pluriactivité en Algérie. La section 3 présente un examen détaillé sur la littérature existante, expose deux théories principales liées à la pluriactivité et propose quatre hypothèses à tester empiriquement. La section 4 présente la méthode d'échantillonnage et la méthodologie de notre travail de recherche. La section 5 présente et discute les résultats de nos enquêtes de terrain. La dernière section offre des remarques finales.

\section{La pluriactivité en Algérie}

A travers le monde, les agriculteurs font recours à de plus en plus aux revenus non agricoles et l'Algérie n'est pas une exception. La pluriactivité en Algérie participe à la survie de l'agriculture Algérienne qui fait face aux pressions exercées par les secteurs non agricoles en compétition et l'accroissement de la contrainte financière. Elle peut donc être considérée comme un moyen de soutien à l'agriculture et à la paysannerie.

En effet, La pluriactivité se perpétue de nos jours comme une nécessité pour une région ou l'emploi est rare et où il est difficile pour les agriculteurs de vivre du seul revenu agricole, comme c'est le cas de l'Algérie.
L'Algérie, comme beaucoup de pays du sud, souffre d'une insuffisance de la production agricole et de la faiblesse de la productivité, ainsi que la dégradation des zones rurales. Afin d'atténuer ces problèmes, la politique agricole en Algérie n'a cessé d'encourager le développement des zones rurales. Depuis 2006, la pluriactivité est encouragée en Algérie, en raison de son importance dans le maintien de la population rurale pauvres et marginalisées, ainsi que dans la création de l'emploi. En particulier, la politique du renouveau rural élaborée en 2006, énonce des buts très ambitieux concernant la pluriactivité en favorisant la diversification des activités et la pluriactivité des ménages (MADR, 2009 ; Chabane, 2013).

La pluriactivité est très répandue à l'échelle internationale. Les enquêtes menées dans plusieurs pays montrent que jusqu'à deux tiers des agriculteurs annoncent avoir un certain nombre d'activités hors agriculture (Kimhi, 2000). Les comparaisons internationales les plus récentes portent sur les pays membres de l'union européenne. Elles sont tirées des «enquêtes structures» communautaires. La dernière entièrement publiée, celle de 2003, donne un taux de pluriactivité des chefs d'exploitation de $27 \%$ en moyenne pour l'Europe (Boudy, 2009).

Pour l'Algérie, les premiers résultats sur la pluriactivité datent de 1980 , ou plus de $75 \%$ des agriculteurs étaient pluriactifs (Chaulet, 1987). Manceron et Aissani (1996) notent que la pluriactivité est la règle pour la moitié environ d'actifs agricoles qui sont occupées moins de six mois dans l'agriculture.

Dans cet article, nous présentons les résultats d'une étude empirique basée sur un échantillon de 270 agriculteurs. Les résultats de notre enquête, montre que sur les 270 exploitants, 151 ou $55,9 \%$ ont confirmés être pluriactifs. Trois destinations dominent les activités secondaires des pluriactifs : la fonction publique $(22,2 \%)$; le commerce $(13 \%)$; et les professions libérales $(8,1 \%)$. La majorité $(65,6 \%)$ des pluriactifs travail dans leur commune, de façon permanente (76,8\%). Plus de $35 \%$ des pluriactifs indiquent que leur principale raison d'être pluriactif et la baisse du revenu agricole. Finalement, 21,9\% des exploitants pluriactifs mentionnent qu'ils l'ont fait par choix. 
En termes de législation, on trouve une absence totale de loi ou de réglementation concernant la pluriactivité. En effet, le secteur informel emploie une proportion importante des pluriactifs. Ces derniers ne bénéficient pas de droits de travail ou d'assurance. Ceci explique pourquoi l'enquête mené par le ministère de l'agriculture et de développement rural a trouvé que seulement $15.9 \%$ des familles agricoles pratique la pluriactivité dans les zones rurales (MADR, 2004). Le manque de législation pousse les exploitants agricoles à travailler d'une façon informelle et non-déclarés.

\section{Revue de la littérature et développement des hypothèses}

La base théorique sur laquelle les travaux antérieurs ont analysé le revenu des agriculteurs est la théorie néoclassique de l'offre de travail (Sumner, 1982 ; Gunter et McNamara, 1990). Selon cette théorie, l'exploitant a le choix entre le travail dans l'exploitation, le travail hors exploitation et les loisirs. L'exploitant maximise son utilité en arrivant à une répartition optimale du temps. L'optimum est atteint lorsque les valeurs marginales du temps consacré à ces trois activités sont égales (Sumner, 1982). Nos hypothèses sont basées sur le modèle théorique de Matshe et Young (2004). Dans ce modèle, l'agriculteur maximise une fonction d'utilité et décide du temps optimal à allouer aux travaux hors ferme. Cette répartition dépend du taux de salaire sur le marché du travail et du salaire de réserve de l'agriculteur (valeur marginale du travail agricole). Le salaire de réserve, à son tour, est déterminé par d'autres variables exogènes, y compris les prix des inputs et des outputs, les caractéristiques de l'exploitation et les caractéristiques des ménages et exploitants agricoles (Matshe et Young, 2004: 177).

Les agriculteurs ont aussi une aversion au risque (Serra et al., 2005). L'un des meilleurs modèles pour considérer le risque de revenu agricole et non agricole est celui d'Andersson et al. (2003). Dans ce modèle, la présence de plusieurs emplois est le résultat d'une optimisation de portefeuille qui prend en compte le revenu agricole et non agricole ainsi que les risques associés à ces revenus. Il faut noter que cette théorie, bien que liée à la théorie de portefeuille utilisé purement dans le domaine des finance (Markowitz, 1958), est basé partiellement sur le comportement de l'agriculteur (pour plus de détails voir Shefrin et Statman, 2000).

Dans cet article, nous avons choisi quatre hypothèses centrales. Ces hypothèses reproduisent les facteurs les plus importants qui poussent les agriculteurs vers la pluriactivité que nous avons identifié dans notre revue littéraire. Bien qu'il existe d'autre hypothèses potentielles, mais nous avons utilisé ces facteurs comme des variable de contrôles.

\subsection{Le Revenu agricole}

Selon le modèle néoclassique, les agriculteurs qui ont des revenus agricoles relativement élevés exigent des salaires plus élevés pour allouer une partie de leur travail au secteur non agricole. D'autre part, le revenu agricole plus élevé réduit le risque de consommation ultérieure pour l'agriculteur parce qu'il peut économiser une partie de son revenu pour une consommation ultérieure.

Sofer (2001) a conclu que l'un des facteurs les plus importants qui stimulent le taux de pluriactivité est la baisse du revenu agricole. Gunter et McNamara (1990, p. 163) ont constaté que le revenu agricole est le facteur le plus important dans la détermination de la probabilité de pluriactivité. Ils ont calculé que la probabilité d'emplois multiples est de $87,1 \%$ pour une exploitation qui a un revenu net de zéro. Cette probabilité diminue à $4,2 \%$ pour une exploitation avec un revenu net de $\$ 40.000$. L'étude de Lass et Gempesaw (1992) sur la Pennsylvanie suggère une conclusion similaire.

Serra et al. (2005), Benjamin (1994) et Woldehanna et al. (2000) confirment que les chutes des prix agricoles entraînent une augmentation de la pluriactivité des ménages agricoles. Matshe et Young (2004) montrent que les agriculteurs du Zimbabwe sont sensibles aux prix des produits agricoles (par rapport aux produits non agricoles). Ils ont constaté qu'une augmentation des prix (relatifs) des produits agricoles aide les agriculteurs à passer moins de temps à l'extérieur de l'exploitation. 
Le risque de revenu a également été considéré comme un facteur important. Serra et al. (2005) montrent qu'une plus grande variabilité du revenu brut agricole augmente la probabilité qu'un ménage agricole soit pluriactif.

Sur la base de la discussion précédente, nous avançons l'hypothèse suivante:

H1 : Tous facteurs étant constant par ailleurs, le revenu agricole est négativement lié à la probabilité que le chef d'exploitation soit pluriactif.

\subsection{L'équipement}

Anseeuw et Laurent (2007) ont constaté que l'accès à un équipement suffisant exige un niveau d'investissement assez important. Cet investissement ne peut être réalisé que progressivement, obligeant les familles agricoles à travailler en dehors de leur exploitation pour accumuler les fonds nécessaires. Donc, selon cette perspective, les ménages qui possèdent déjà de grandes installations ont moins besoin de travailler à l'extérieur de leurs exploitations.

Selon le modèle d'Andersson et al. (2003), si les ménages agricoles ne peuvent pas diversifier leurs investissements, l'avantage de la pluriactivité en termes de réduction des risques devient très important. Par conséquent, les agriculteurs mieux équipés ont une plus grande capacité à diversifier leur production, réduisant ainsi leur risque de revenu. Par conséquent, les agriculteurs ayant de meilleurs équipements n'ont pas besoin de travailler hors ferme afin de réduire le risque de leur revenu total.

Le modèle de Ahituv et Kimhi (2002) prédit un effet similaire. Plus précisément, l'utilisation de technologies avancées augmente le revenu agricole et, par conséquent, réduit le nombre d'agriculteurs pluriactifs.

Ces trois études sont unanimes sur l'effet du matériel agricole sur le revenu (effet positif), sur le risque de revenu (effet négatif) et donc sur la pluriactivité (effet négatif). Nous proposons l'hypothèse suivante:

H2 : Tous facteurs étant constants par ailleurs, la disponibilité de l'équipement agricole dans l'exploitation réduit la probabilité que le chef d'exploitation soit pluriactif.

\subsection{La situation financière des ménages}

La situation financière des ménages agricoles est liée au modèle néoclassique ainsi qu'à la théorie du portefeuille. D’une part, les ménages pauvres ont un faible salaire de réserve et sont donc plus susceptibles d'accepter un travail en dehors de l'agriculture. D'autre part les ménages pauvres sont plus sensibles aux risques liés à la faiblesse de leur revenu, pour réduire le risque de revenu, les agriculteurs doivent diversifier leurs revenus en acceptant du travail non agricole.

Selon Reardon et al. (1992), la théorie du portefeuille a des implications contradictoires. D'une part, les ménages qui ont des ressources suffisantes sont moins sensibles au risque de revenu et peuvent donc se permettre de réaliser des investissements non agricoles. Dans le même temps, les ménages à faible revenu sont également obligés de diversifier leurs emplois afin de réduire le risque de leur revenu. Les mêmes auteurs rapportent que la preuve empirique est ambiguë.

Edmond et Crabtree (1994) ont conclu que les ménages à faible revenu agissent comme des facteurs incitatifs, c'est-à-dire en poussant les agriculteurs à accepter des travaux hors ferme. Plusieurs études indiquent que les ménages ayant un revenu supplémentaire sont moins susceptibles d'être pluriactifs (Tavernier et al., 1997; Serra et al., 2005; Lass et Gempesaw, 1992; Matshe et Young, 2004).

Sur la base des résultats empiriques mentionnés ci-dessus, nous avançons l'hypothèse suivante:

H3 : La bonne situation financière du ménage agricole a un impact négatif sur la probabilité du choix de pluriactivité par le chef d'exploitation.

\subsection{La taille de l'exploitation}

Simpson et Kapitany (1983) trouvent que le capital est le facteur le plus important qui réduit la probabilité de pluriactivité. Serra et al. (2005) montrent que les chefs des grandes fermes sont beaucoup moins susceptibles d'avoir un travail hors ferme. Des conclusions similaires ont été tirées chez Alasia et al. (2009), Ben- 
jamin et Kimhi (2006) et Deininger et Olinto (2001). Matshe et Young (2004) ont constaté que la taille des terres arables disponibles pour le ménage est une caractéristique qui a un impact important sur leur décision de répartition $\mathrm{du}$ travail. Les agriculteurs des petites exploitations sont plus susceptibles d'être pluriactifs et de travailler plus d'heures hors ferme. Ils concluent que ces agriculteurs sont obligés de réduire leurs salaires de réserve pour les travaux non agricoles en raison du travail limité offert par leurs exploitations.

D'autres études ont abouti à la même conclusion. Il s'agit notamment de Mishra et Mohart (2001) Mishra et Goodwin (1997) Corsi et Salvioni (2012) et Bojnec et al. (2003). Cependant, nous avons trouvé deux études où l'effet de la taille de l'exploitation n'a pas d'effet significatif: Abdulai et Delgado (1999) et Kimhi et Rapaport (2004).

Malgré la diversité des résultats, nous constatons que la majorité des recherches indiquent un effet négatif de la taille de l'exploitation sur la probabilité de la pratique de la pluriactivité. Nous proposons donc l'hypothèse suivante:

H4 : La taille de l'exploitation a un effet négatif sur la probabilité du choix de pluriactivité par le chef d'exploitation.

\subsection{Les variables de contrôle}

La pluriactivité est un phénomène complexe, ce qui entraine le fait que les variables associées avec nos hypothèses pourraient bien être insuffisantes à expliquer le choix de la pluriactivité chez les exploitants algériens. Nous ajoutons donc six variables de contrôle pour compléter notre modèle empirique et assurer la viabilité statistique de notre modèle économétrique.

\section{Géographie et topographie}

L'importance du revenu hors ferme varie selon la région et il est très sensible à la structure de l'économie locale (Hearn et al., 1996). Étant donné que les salaires de réserve sont plus ou moins homogènes sur l'ensemble du pays, il est possible que les salaires offerts sur les marchés du travail des différentes provinces aient un effet sur la décision d'être pluriactif. Aussi, la pluriac- tivité peut être différente selon les types de topographie pour des raisons socio-économiques, d'infrastructure et de distance des centres économiques (Liu et al. 2013; Sofer, 2001; Edmond et Crabtree, 1994; Kimhi et Rapaport, 2004).

\section{La taille du ménage}

Zhao (2014) a constaté que les grands ménages ont une plus grande motivation pour trouver des formes de diversification de leurs revenus. Brosig et al. (2009) et Deininger et Olinto (2001) ont confirmé que les agriculteurs aux familles nombreuses ont une plus grande tendance à être pluriactifs. Cependant, Abdulai et Delgado (1999) et Lass et Gempesaw (1992) n'ont trouvé aucune relation significative entre la pluriactivité et la taille du ménage de l'exploitant.

\section{Le bétail}

Deininger et Olinto (2001) et Lass et Gempesaw (1992) montrent que les agriculteurs avec un grand nombre de têtes de bétail ont une faible tendance à être pluriactifs. Serra et al. (2005) expliquent que les vaches laitières dans les fermes nécessitent beaucoup de main-d'œuvre et peuvent avoir un effet significatif et négatif sur la probabilité de pluriactivité. Cependant, Abdulai et Delgado (1999) et Corsi et Salvioni (2012) en Italie ne trouvent aucun effet significatif.

\section{L'expérience agricole}

Simpson et Kapitany (1983: 801) affirment que les agriculteurs permanents sont moins enclins à s'engager dans un travail hors ferme. Leurs résultats montrent que l'expérience de l'exploitant a un impact significatif et négatif sur la probabilité d'être pluriactif. Pour les agriculteurs âgés, Mishra et Goodwin (1997) et Benjamin et Kimhi (2006) ont trouvé que les années d'expériences dans l'exploitation réduisent la probabilité d'un travail hors ferme. Tavernier et al. (1997) ont estimé qu'un agriculteur qui possède $10 \%$ de plus d'expérience était $11 \%$ moins susceptible d'être pluriactif.

\section{L'âge}

Selon l'hypothèse du cycle de vie de Gould et Saupe (1989), les travailleurs âgés ont tendance à consommer ce qu'ils ont accumulé pendant 
leur jeunesse. L'âge est également un indicateur pour la composante «expérience» du capital humain (Gunter et McNamara, 1990). Cependant, la majorité des études précédentes ont principalement trouvé un effet non linéaire de l'âge sur la pluriactivité. Sumner (1982), Huffman (1980) et Lass et Gempesaw (1992) ont utilisé des termes linéaires et quadratiques pour représenter cet effet non-linéaire de l'âge.

\section{Le capital humain}

Huffman (1980) soutient que l'effet net du niveau d'instruction sur l'emploi hors exploitation est ambigu. En principe, le niveau d'instruction augmente le salaire hors ferme. Pour une productivité agricole fixe, l'effet total est clair : des niveaux d'instruction plus élevés conduisent à une plus grande incidence sur la pluriactivité. Cependant, le niveau d'instruction augmente également la productivité des agriculteurs, et peut donc avoir l'effet inverse. Par conséquent, l'effet global peut aller dans l'un ou l'autre sens. Cependant, la grande majorité de la littérature a trouvé un effet positif (Matshe et Young, 2004; Bojnec et al., 2003; Martinez Jr et al., 2014; Alasia et al., 2009; Liu et al., 2013; Brosig et al., 2009; et Benjamin et Kimhi, 2006).

\section{Echantillon et méthode}

\subsection{L'échantillonnage}

Nous avons choisi de travailler sur trois provinces situées au centre de l'Algérie : Blida, Médéa et Djelfa couvrant le nord, le milieu et le sud du pays. Pour chaque province, nous avons choisi trois municipalités (une en plaine, une en piémont, et une en montagne). La taille de notre échantillon est imposée par nos ressources financières limitées. Nous avons donc choisi, au hasard, 30 exploitations de chaque commune, 90 exploitations de chaque province, ce qui a donné au total 270 exploitations.

Pour des raisons bureaucratiques, nous n'avons pas eu accès à la liste complète des agriculteurs des communes choisies. Des employés des directions des services agricoles des trois provinces ont tiré au hasard le nombre demandé d'exploitants. Nous avons ensuite contacté ces agriculteurs pluriactifs directement.
Bien que notre échantillonnage au niveau de chaque municipalité soit aléatoire, notre échantillon est basé sur un mélange délibéré de quotas et d'échantillonnage aux niveaux provincial et régional. Notre choix de quotas de 30 exploitations par commune et 3 communes par province dépendait à la fois de nos ressources limitées et de notre objectif d'avoir un mélange équilibré de proximité avec la capitale Alger, ainsi qu'un mélange équilibré avec les trois topographies (plaine, piémont et montagne).

Notre choix de la partie centrale du pays repose sur trois considérations. D'abord, elle est représentative des parties Est et Ouest du pays sur les plans du relief et du type d'agriculture. Deuxièmement, il nous fallait des lieux d'enquête aisés à atteindre par rapport à notre résidence. En effet, obtenir des taux de réponse élevés par des moyens autres que le contact personnel s'avérait impossible. Même les contacts personnels nécessitaient de longues négociations et des recommandations des autorités locales. La tentative d'élargir notre échantillon à d'autres régions s'est révélée impossible. Troisièmement, parmi nos variables de contrôle se trouve la proximité des centres économiques importants et la topographie comme caractéristiques qui influencent la pluriactivité. Nous avons donc besoin de sélectionner des zones et des régions qui correspondent à nos objectifs de recherche en obtenant un échantillon avec la distance et les dimensions de la topographie.

Dans l'ensemble, nous pensons qu'un mélange délibéré d'échantillonnage et de quotas correspond à nos objectifs de recherche.

\subsection{Le questionnaire}

Un questionnaire détaillé a été administré aux agriculteurs afin d'en recueillir des informations détaillées sur le ménage et l'exploitation agricole. Nous avons recueilli des informations sur le genre, l'état matrimonial, l'âge, l'expérience en agriculture, le niveau d'instruction (6 niveaux), les qualifications (4 niveaux), le nombre de garçons, les filles et les personnes qui ont quitté le ménage, le nombre de chambres, la superficie totale des terres cultivées, les superficies irriguées, la localisation géographique (plaine, piémont, montagne). 
Les questions dichotomiques étaient les suivantes: si le fermier est propriétaire d'une voiture, d'un camion, d'une fourgonnette, d'un équipement de traction, d'un matériel d'irrigation, de bétail, si le ménage de l'agriculteur a une consommation suffisante, des vêtements, si la ferme possède une production suffisante et des gains suffisants.

\subsection{Analyse en composantes principales}

Dans notre questionnaire, nous avons posé des questions multiples pour couvrir certaines caractéristiques ou facteurs. Nous extrayons ces caractéristiques communes à l'aide de l'analyse en composantes principales (ACP). Dans l'ACP, l'objectif est d'extraire un ensemble de variables $\mathrm{p}$, un ensemble réduit de $\mathrm{m}$ composantes qui représentent la plus grande proportion de la variance dans les variables p. En d'autres termes, nous voulons réduire un ensemble de mesures ou de variables $\mathrm{p}$ à un ensemble de $\mathrm{m}$ dimensions sous-jacentes.

L'ACP peut se faire simplement pour réduire un ensemble de variables $\mathrm{p}$ à $\mathrm{m}$ composantes (ou facteurs) avant qu'une analyse complémentaire de ces facteurs ne puisse être effectuée. Par exemple, nous avons quatre questions (éléments ou variables) dans lesquelles nous demandons si l'agriculteur possède un camion, une camionnette, un équipement de traction et des équipements d'irrigation. Ces quatre variables représentent un facteur unique que l'on appelle «facteur d'équipement». Ce facteur sera ensuite utilisé comme prédicteur dans un modèle de probabilité (pour plus de détails, voir Carricano et al., 2010).

\subsection{Modèles de probabilité}

Notre variable dépendante est catégorique, de sorte que les modèles de probabilité, tels que le probit, sont les modèles les plus appropriés pour estimer les relations et faire des inférences. Ces modèles sont largement décrits dans la littérature statistique (voir, par exemple, Greene, 2010 ; Carricano et al., 2010 et Pétry, 2003). Dans ces modèles, on cherche à estimer la probabilité qu'un agriculteur soit pluriactif, c'est- à-dire $P(Y=1)$ où l'événement $Y=1$ signifie que l'agriculteur est pluriactif. La relation entre cette probabilité et les variables explicatives définit le modèle. Plus précisément,

$$
P(Y=1)=\Phi\left(\alpha_{0}+\alpha_{1} Z_{1}+\alpha_{2} Z_{2}+\ldots+\alpha_{k} Z_{k}\right)
$$

Où les $\alpha$ sont des coefficients à estimer, les $Z$ sont des variables explicatives, et la fonction $\Phi(x)$ représente la distribution normale cumulative,

$$
\Phi(x)=\frac{1}{\sqrt{2 \pi}} \int_{-\infty}^{x} e^{-t^{2}} d t
$$

Lorsqu'il y a plus de deux catégories, on peut utiliser le modèle ordonné. Supposons qu'il y ait $\mathrm{N}$ catégories avec les probabilités $P(Y=1)$, $P(Y=2), \ldots, P(Y=\mathrm{N})$. Ces probabilités sont données par

$$
\begin{gathered}
P\left(Y_{i}=1\right)=\Phi\left(\mu_{1}-I_{i}\right) \\
P\left(Y_{i}=2\right)=\Phi\left(\mu_{2}-I_{i}\right)-\Phi\left(\mu_{1}-I_{i}\right) \\
P\left(Y_{i}=j\right)=\Phi\left(\mu_{j}-I_{i}\right)-\Phi\left(\mu_{j-1}-I_{i}\right) \\
P\left(Y_{i}=N\right)=1-\Phi\left(\mu_{N-1}-I_{i}\right)
\end{gathered}
$$$$
\text { où } I_{i}=\alpha_{0}+\alpha_{1} Z_{1}+\alpha_{2} Z_{2}+\ldots+\alpha_{k} Z_{k} \text {. }
$$

\section{Résultats}

\subsection{Statistiques descriptives}

Le Tableau 1 donne quelques statistiques de base pour les variables continues (variables qui ne peuvent pas être résumées par les fréquences).

L'âge moyen est relativement élevé ; avec une moyenne de 53,81 ans, le niveau de vieillissement des agriculteurs est relativement important. Il y a beaucoup plus d'agriculteurs âgés que de jeunes, comme l'indique l'écart-type, ce qui suggère qu'environ $95 \%$ de la population des agriculteurs a entre 28 et 80 ans $(53,81 \pm 2 \times 12,99)$. Les ménages sont généralement de grandes tailles avec une moyenne de 6,71 membres par ménage, mais il existe une taille extrême allant jusqu'à 18 membres. La taille moyenne de la maison est d'environ 4 chambres (mais varie entre 1 et 14 chambres). 
Tableau 1 - Statistiques descriptives.

\begin{tabular}{|l|c|c|c|c|}
\hline & $\begin{array}{c}\text { Mini- } \\
\text { mum }\end{array}$ & $\begin{array}{c}\text { Maxi- } \\
\text { mum }\end{array}$ & Moyenne & $\begin{array}{c}\text { Ecart } \\
\text { Type }\end{array}$ \\
\hline Age & 18 & 90 & 53.81 & 12.99 \\
\hline $\begin{array}{l}\text { Nombre de } \\
\text { personnes } \\
\text { vivantes dans } \\
\text { le foyer }\end{array}$ & 1 & 18 & 6.71 & 2.63 \\
\hline $\begin{array}{l}\text { Nombre de } \\
\text { chambres }\end{array}$ & 1 & 14 & 4.03 & 1.98 \\
\hline $\begin{array}{l}\text { Surface } \\
\text { Agricole } \\
\text { Utile (SAU) }\end{array}$ & 0 & 250 & 15.72 & 23.69 \\
\hline $\begin{array}{l}\text { Surface } \\
\text { irriguée }\end{array}$ & 0 & 95 & 5.26 & 9.49 \\
\hline
\end{tabular}

$N=270$.

Il existe une grande variabilité dans la taille de l'exploitation (SAU). Bien que la taille moyenne de l'échantillon soit de 15,72 ha, l'écart type est de 23,69 ha, ce qui indique l'existence d'exploitations de dimension relativement importantes. En revanche, les surfaces irriguées dans ces exploitations sont beaucoup plus petites, avec une moyenne de 5,26 ha en moyenne. Notre échantillon est dominé par les agriculteurs mariés $(92,6 \%)$ et de sexe masculin $(98,5 \%)$.

Sur les 270 agriculteurs, $151(55,9 \%)$ ont confirmé qu'ils étaient pluriactifs. La majorité $(59,6 \%)$ des 151 agriculteurs pluriactifs ont indiqué que la majeure partie de leur revenu provient de l'extérieur de l'exploitation.

\subsection{Analyse factorielle}

Nos variables explicatives sont potentiellement fortement corrélées et peuvent donc générer des problèmes de multicollinéarité. Nous utilisons la méthode des composantes principales pour réduire le nombre de variables à un niveau maitrisable.

Le Tableau 2 présente les résultats d'une analyse en composantes principales pour cinq groupes de variables représentant le capital humain du chef de ménage, la taille du ménage, la situation financière du ménage, l'équipement agricole et enfin le revenu agricole. Pour le capital humain de l'exploitant, nous avons recueilli deux informations sur le niveau d'instruction et les qualifications. Les estimations fournissent deux composantes, mais une seule composante (ou facteur) a une valeur propre supérieure à 1 . Selon la règle de Kaiser (voir Carricano et al., 2010), les facteurs dont les valeurs propres sont inférieures à 1 ne sont pas significatifs. Les deux variables (instruction et diplôme) sont représentées de manière adéquate par un facteur unique représentant plus de $77 \%$ de la variance des deux variables originales. Ces deux variables sont fortement corrélées, ce qui explique leurs charges égales. Nous avons extrait ce facteur en tant que variable explicative.

Pour la taille du ménage, nous avons 3 variables de base : le nombre de garçons, le nombre de filles et le nombre de personnes qui ont quitté le ménage. Un facteur expliquant $42,48 \%$ de la variance de trois variables est important (valeur propre $=1,275$ ). Les deux autres facteurs ont des valeurs propres inférieures à 1 .

La situation financière des ménages est mesurée par quatre variables: le nombre de chambres; la présence d'une voiture et la couverture des besoins en nourriture et en vêtements. L'ampleur de ces variables reflète le bien-être du ménage. Un facteur expliquant plus de $50 \%$ de la variation des quatre mesures est important avec une valeur propre importante de plus de 2 .

Les deux derniers facteurs sont extraits de la même manière. Le facteur «équipement» reflète la disponibilité des moyens de production, tandis que le facteur «revenu» reflète la rentabilité de l'exploitation. Les cinq facteurs ont été extraits par SPSS en utilisant la méthode de régression. Ces variables sont normalisées avec une moyenne de zéro et une variance unitaire.

\subsection{Résultats du modèle de probabilité de la pluriactivité}

Le Tableau 3 présente les résultats de l'estimation d'un modèle probit qui explique la probabilité qu'un agriculteur soit actuellement pluriactif. La variable dépendante est binaire (pluriactive $=1$; non pluriactive $=0$ ), et les variables indépendantes sont un mélange de variables binaires (muettes) et de variables d'échelle. 
Tableau 2 - Analyse Factorielle et Extraction des Cinq Facteurs.

\begin{tabular}{|c|c|c|c|c|c|}
\hline Composantes & $\begin{array}{c}\text { Valeurs } \\
\text { Propres } \\
\text { (Eigenvalues) }\end{array}$ & $\begin{array}{c}\% \\
\text { Variance }\end{array}$ & $\begin{array}{c}\% \\
\text { Cumulative }\end{array}$ & $\begin{array}{l}\text { Variables } \\
\text { (Items) }\end{array}$ & $\begin{array}{l}\text { Charge } \\
\text { (Loading) }\end{array}$ \\
\hline \multicolumn{6}{|c|}{ Facteur: Compétence Technique } \\
\hline 1 & 1,54 & 77,01 & 77,01 & Niveau d'instruction & 0,87 \\
\hline 2 & 0,46 & 22,98 & 100,00 & Diplôme & 0,87 \\
\hline \multicolumn{6}{|c|}{ Facteur : Taille du Ménage } \\
\hline 1 & 1,27 & 42,48 & 42,48 & Nombre de garçons & 0,71 \\
\hline 2 & 0,91 & 30,37 & 72,86 & Nombre de filles & 0,63 \\
\hline 3 & 0,81 & 27,13 & 100,00 & $\begin{array}{l}\text { Nombre de personnes } \\
\text { qui ont quitté le foyer }\end{array}$ & 0,60 \\
\hline \multicolumn{6}{|c|}{ Facteur : Situation Financière } \\
\hline 1 & 2,09 & 52,41 & 52,41 & Nombre de chambres & 0,38 \\
\hline 2 & 0,99 & 24,86 & 77,28 & Possession de voiture & 0,45 \\
\hline 3 & 0,82 & 20,53 & 97,82 & $\begin{array}{l}\text { Consommation suff- } \\
\text { isante }\end{array}$ & 0,93 \\
\hline 4 & 0,08 & 2,18 & 100,00 & Besoins vestimentaires & 0,93 \\
\hline \multicolumn{6}{|c|}{ Facteur : Equipement } \\
\hline 1 & 1,41 & 35,45 & 35,45 & Camion & 0,52 \\
\hline 2 & 0,98 & 24,66 & 60,12 & Camionnette & 0,65 \\
\hline 3 & 0,90 & 22,56 & 82,68 & Matériel de traction & 0,62 \\
\hline 4 & 0,69 & 17,31 & 100,00 & Equipement d'irrigation & 0,57 \\
\hline \multicolumn{6}{|c|}{ Facteur : Revenu Exploitation } \\
\hline 1 & 1,59 & 79,96 & 79,96 & Production suffisante & 0,89 \\
\hline 2 & 0,40 & 20,03 & 100,00 & Revenu suffisant & 0,89 \\
\hline
\end{tabular}

Source : Résultats de l'enquête.

Les variables de contrôle binaires incluent: (I) Deux variables muettes de la province pour Blida et Djelfa, qui sont contrastés avec Médéa ; (II) Deux variables muettes topographiques, la montagne et le piémont, qui sont contrastés avec la plaine ; (III) La variable muette représentant le «bétail» est égal à 1 si l'opérateur déclare avoir du bétail et zéro sinon.

Les autres variables de contrôle d'échelle comprennent : (I) deux premières variables représentées par l'âge et l'âge au carré ; (II) le capital humain, (III) la taille du ménage, et (IV) l'expérience.

Nous avons enfin quatre variables qui seront utilisées pour tester nos hypothèses, notamment la situation financière des ménages, l'équipe- ment, le revenu agricole et la taille de l'exploitation.

La taille de l'exploitation est mesurée par la superficie agricole totale (SAT). Étant donné que les tailles des exploitations ne sont pas semblables d'une province à l'autre (la province du sud a des tailles beaucoup plus grandes), nous avons décidé d'utiliser trois termes d'interaction entre le SAT et la variable binaire représentant chaque province. Cette spécification nous aide à saisir l'effet de la taille qui sera spécifique à la province en question. La superficie irriguée et l'expérience sont les deux dernières variables.

L'effet topographique est quelque peu surprenant puisque les agriculteurs de montagne ont la même probabilité que ceux de la plaine. 
Tableau 3 - Résultats du modèle probit simple.

\begin{tabular}{|c|c|c|c|c|c|}
\hline & \multirow[b]{2}{*}{ Coefficient } & \multirow{2}{*}{$\begin{array}{c}\text { Ecart } \\
\text { Type }\end{array}$} & \multicolumn{2}{|c|}{ Test d'hypothèse } \\
\hline & & & & $\begin{array}{c}\text { Wald } \\
\text { Chi-Deux }\end{array}$ & Valeur $p$ \\
\hline \multicolumn{6}{|l|}{ Variables de contrôles } \\
\hline Intercepte & & 3.441 & 1.51 & 5.16 & 0,023 \\
\hline Topographie : Montagne & & 0.258 & 0.24 & 1.14 & 0.285 \\
\hline Topographie : Piémont & & 1.082 & 0.27 & 15.58 & 0.000 \\
\hline Province : Blida & & -0.181 & 0.39 & 0.21 & 0.646 \\
\hline Province : Djelfa & & 0.359 & 0.45 & 0.61 & 0.433 \\
\hline Facteur : Taille du Ménage & & 0.025 & 0.11 & 0.04 & 0.833 \\
\hline Facteur : Capital Humain & & 0.401 & 0.12 & 9.74 & 0.002 \\
\hline Age & & 0.077 & 0.05 & 2.22 & 0.136 \\
\hline $\mathrm{Age}^{2}$ & & -0.001 & 0.01 & 3.40 & 0.065 \\
\hline Bétail & & -0.044 & 0.22 & 0.04 & 0.841 \\
\hline Expérience & & 0.044 & 0.09 & 0.22 & 0.641 \\
\hline \multicolumn{6}{|l|}{ Variables d'hypothèses } \\
\hline Facteur : Revenu Exploitation & $\mathrm{H} 1$ & 0.024 & 0.11 & 0.05 & 0.825 \\
\hline Facteur : Equipment & $\mathrm{H} 2$ & -0.345 & 0.11 & 9.11 & 0.003 \\
\hline Facteur : Situation Financière Men. & $\mathrm{H} 3$ & -0.119 & 0.11 & 1.16 & 0.282 \\
\hline SAU $\times$ Blida & $\mathrm{H} 4$ & 0.000 & 0.01 & 0.00 & 0.993 \\
\hline SAU $\times$ Médéa & $\mathrm{H} 4$ & 0.011 & 0.01 & 0.86 & 0.353 \\
\hline SAU $\times$ Djelfa & $\mathrm{H} 4$ & 0.011 & 0.01 & 2.64 & 0.104 \\
\hline Surface Irriguée & $\mathrm{H} 4$ & -0.027 & 0.01 & 4.31 & 0.038 \\
\hline
\end{tabular}

Notes: Le modèle estimé est P(Pluriactive) $=\Phi\left(I_{i}\right)$, lorsque $I_{i}=\alpha_{0}+\alpha_{1} Z_{1}+\alpha_{2} Z_{2}+\ldots+\alpha_{k} Z_{k}$, et $\Phi($. représente la densité cumulative normale. Les provinces de Blida et Djelfa sont contrastées avec la wilaya de Médéa. La Montagne et le Piémont sont contrastés avec la Plaine. La SAU est la surface agricole utile.

Cependant, les agriculteurs des piémonts sont nettement plus susceptibles d'être pluriactifs (le coefficient est égal à 1,082 est très significatif avec une valeur $\mathrm{p}<0,001)$. Les deux coefficients des provinces sont insignifiants, ce qui suggère l'homogénéité des trois provinces étudiées.

La taille du ménage a un coefficient proche de zéro et insignifiant, signifiant que la taille du ménage agricole n'influence pas la décision de pluriactivité.

Le facteur «capital humain» est le plus influent parmi les cinq facteurs. Le coefficient associé à ce facteur est le plus grand en valeur absolue. Le coefficient est positif $(0,401)$ et très significatif (valeur $\mathrm{p}=0,002$ ) et implique que les compétences d'un agriculteur augmentent de manière significative la probabilité qu'il soit pluriactif.

Le coefficient de l'âge est positif (0.077), mais faiblement significatif (valeur $\mathrm{p}=0.136$ ). Cependant, le terme quadratique ( $\left(\hat{g} g e^{2}\right.$ ) est négatif comme prévu et raisonnablement significatif (valeur $\mathrm{p}=0,065)$. La valeur du coefficient apparaît très faible (-0.001), mais son effet demeure néanmoins significatif. Il est clair que l'effet de l'âge augmente avec le jeune âge. En fait, entre 18 et 39 ans, l'effet est positif. Ensuite (à partir de 40 ans), cet effet devient négatif.

Le coefficient de la variable muette du bétail est négatif et significatif. Donc la présence du 
bétail dans l'exploitation influence négativement et fortement la probabilité de pluriactivité. Enfin, l'expérience n'a pas d'influence sur la pluriactivité, probablement à cause de la forte corrélation entre l'expérience d'une part et l'âge et le capital humain de l'autre.

La première hypothèse ( $\mathrm{H} 1$ : le revenu agricole est négativement lié à la probabilité de la pluriactivité) est rejeté, car le facteur «revenu» n'a pas de rôle explicatif sur la probabilité que l'agriculteur soit pluriactif (coefficient $=0,024$, valeur $\mathrm{p}=0,825$ ).

En revanche, notre deuxième hypothèse est confirmée (H2: plus d'équipement réduit la probabilité de pluriactivité). Le coefficient du facteur «équipement» est négatif $(-0,345)$ et très significatif (valeur $p=0,003$ ), ce qui implique que l'utilisation de l'équipement réduit la probabilité que l'exploitation soit pluriactive.

La troisième hypothèse n'est pas acceptée. Le facteur «situation financière» du ménage agricole n'a pas d'impact significatif sur la probabilité de la pluriactivité.

Enfin, il existe un soutien partiel pour la quatrième hypothèse. La taille de l'exploitation est représentée par l'interaction de la variable surface agricole utile (SAU) avec trois variables muettes des provinces. Il est également représenté par la surface irriguée. Toutes les variables d'interaction SAU sont négligeables, suggérant que la taille de l'exploitation n'a aucun impact sur la probabilité de la pluriactivité, indépendamment de la distance des centres économiques. Néanmoins, l'hypothèse ne peut pas être rejetée car la variable de surface irriguée est significative et a un coefficient négatif $(-0,027)$ avec une valeur $\mathrm{p}$ égale à 0,038 . Donc, pour la taille, seule la zone irriguée réduit la probabilité de pluriactivité.

\subsection{Contrôles de robustesse}

Les résultats ci-dessus sont basés sur l'hypothèse que la pluriactivité est un choix binaire, c'est-à-dire que l'opérateur peut être pluriactif ou mono-actif. Dans cette section, nous étendons cette contrainte en supposant qu'il existe deux niveaux d'activités multiples: secondaire (modérée) et principale (intensive). La variable dépendante est définie à trois niveaux (non-pluriactif $=0$; revenu agricole pluriactif modéré avec plus de $50 \%=1$; pluriactif avec un revenu agricole intensif inférieure à $50 \%=2$ ). Les résultats sont présentés par le Tableau 4.

Ce type de variable dépendante limitée est modélisé de manière adéquate par le modèle de probit ordonné qui explique la probabilité qu'un agriculteur soit modérément à très pluriactif. Les deux coefficients de seuil, $\mu_{1}$ et $\mu_{2}$ sont significatifs aux niveaux de $10 \%$ et $5 \%$ respectivement, ce qui indique l'existence de deux niveaux de pluriactivité. Les résultats sont très semblables à ceux du probit simple. En général, les mêmes conclusions restent valides.

En ce qui concerne les hypothèses, le modèle ordinal est conforme au modèle simple. Les hypothèses 1 et 3 sont rejetées, l'hypothèse 2 est fortement confirmée, tandis que l'hypothèse 4 est partiellement confirmée.

La première hypothèse (revenu agricole) a également été rejetée malgré la signifiance du coefficient associé à cette variable. La raison de ce rejet est que le coefficient est positif, suggérant que le revenu agricole augmente la probabilité d'activités multiples, contrairement à la présomption de l'hypothèse $\mathrm{H} 1$.

\section{Conclusions}

Cet article visait à tester empiriquement quatre hypothèses relatives à la pluriactivité et tirées du modèle néoclassique et de la théorie du portefeuille. Nous avons effectué des tests formels de nos hypothèses en utilisant des modèles de probabilité.

Nous n'avons pas pu confirmer l'effet négatif du revenu agricole, contrairement aux résultats d'études antérieures. Cette incohérence avec les résultats antérieurs peut être due à la possibilité que le revenu agricole soit fortement corrélé avec le matériel agricole. Ce dernier est très important dans notre étude et c'est probablement la raison pour laquelle le revenu agricole est négligeable. En effet, toutes les études ci-dessus qui ont confirmé le revenu agricole comme facteur important n'incluent pas l'équipement en tant que cofacteur dans leur analyse. Ce résultat souligne l'importance de la spécification du modèle 
Tableau 4 - Résultats du modèle probit ordinal.

\begin{tabular}{|c|c|c|c|c|c|}
\hline & \multirow[b]{2}{*}{ Coefficient } & \multirow{2}{*}{$\begin{array}{l}\text { Ecart } \\
\text { Type }\end{array}$} & \multicolumn{2}{|c|}{ Teste d'hypothèse } \\
\hline & & & & $\begin{array}{c}\text { Wald } \\
\text { Chi-Deux }\end{array}$ & Valeur $p$ \\
\hline \multicolumn{6}{|l|}{ Variables de contrôles } \\
\hline Coefficient Seuil $\mu_{1}$ & & 2.323 & 1.3355 & 3.027 & 0.082 \\
\hline Coefficient Seuil $\mu_{2}$ & & 3.157 & 1.3398 & 5.553 & 0.018 \\
\hline Topographie : Montagne & & 0.209 & 0.2193 & 0.909 & 0.340 \\
\hline Topographie : Piémont & & 1.101 & 0.2358 & 21.791 & 0.000 \\
\hline Province : Blida & & -0.142 & 0.3389 & 0.177 & 0.674 \\
\hline Province : Djelfa & & 0.401 & 0.3695 & 1.181 & 0.277 \\
\hline Facteur : Taille du Ménage & & 0.022 & 0.1009 & 0.048 & 0.826 \\
\hline Facteur : Capital Humain & & 0.287 & 0.1052 & 7.453 & 0.006 \\
\hline Age & & 0.055 & 0.0455 & 1.454 & 0.228 \\
\hline $\mathrm{Age}^{2}$ & & -0.001 & 0.0004 & 2.410 & 0.121 \\
\hline Bétail & & -0.136 & 0.1953 & 0.486 & 0.486 \\
\hline Expérience & & 0.034 & 0.0724 & 0.217 & 0.641 \\
\hline \multicolumn{6}{|l|}{ Variables d'hypothèses } \\
\hline Facteur : Revenu Exploitation & H1 & 0.224 & 0.0948 & 5.600 & 0.018 \\
\hline Facteur : Equipement & $\mathrm{H} 2$ & -0.306 & 0.1018 & 9.052 & 0.003 \\
\hline Facteur : Situation Financière Men. & H3 & -0.048 & 0.0945 & 0.262 & 0.609 \\
\hline SAU $\times$ Blida & $\mathrm{H} 4$ & 0.006 & 0.0098 & 0.327 & 0.567 \\
\hline SAU $\times$ Médéa & H4 & 0.006 & 0.0103 & 0.349 & 0.554 \\
\hline SAU $\times$ Djelfa & $\mathrm{H} 4$ & 0.007 & 0.0041 & 2.584 & 0.108 \\
\hline Surface Irriguée & $\mathrm{H} 4$ & -0.032 & 0.0125 & 6.428 & 0.011 \\
\hline
\end{tabular}

Notes: Le modèle estimé est le probit ordinal décrit dans la section 3. Les provinces de Blida et Djelfa sont contrastées avec la wilaya de Médéa. La Montagne et le Piémont sont contrastés avec la Plaine. La SAU est la surface agricole utile.

dans l'étude de la pluriactivité. L'équipement agricole (notre deuxième hypothèse) semble fournir une plus grande pertinence de l'information que le revenu agricole car il englobe les revenus mais fournit un signal supplémentaire sur la productivité, l'intensité du travail, le revenu et la rentabilité de l'exploitation. L'ignorance de l'équipement agricole a probablement conduit à un modèle de spécification incorrecte dans les études précédentes.

Comme le revenu agricole, la situation financière du ménage (troisième hypothèse) n'est pas importante. Ceci est aussi contraire aux résultats des études précédentes. Enfin, notre quatrième hypothèse (la taille de l'exploitation) est partiellement soutenue. Seule la superficie irriguée est significative et avec l'effet négatif attendu. Le fait que la superficie totale en Algérie n'est pas importante reflète la nature semi-aride des terres agricoles en Algérie.

Dans cet article, nous avons démontré que la pluriactivité est un phénomène complexe qui doit être étudié de manière exhaustive. Des études antérieures ont examiné certains facteurs potentiels mais ont négligé d'autres. Nous avons recueilli des données sur tous les facteurs potentiels que 
nous avons trouvés dans la littérature et les modèles estimés qui prédisent la probabilité qu'un agriculteur particulier adopte une pluriactivité en utilisant des spécifications complètes plutôt que partielles dans la littérature. Nos résultats suggèrent que plusieurs facteurs fondamentaux contribuent à la détermination du choix des agriculteurs du travail hors ferme. Certains d'entre eux semblent être universels, tandis que d'autres semblent être spécifiques au pays ou/et à sa culture (locales). Notre étude doit donc être complétée par d'autres études supplémentaires utilisant les données d'autres pays en développement afin de mieux comprendre ce phénomène important.

Bien que le contexte touche l'agriculteur algérien, nous croyons que des enseignements peuvent être tirés pour de nombreux pays en développement, en particulier ceux qui partagent des caractéristiques communes avec l'Algérie.

La politique agricole en Algérie veut encourager la pluriactivité pour permettre à l'agriculteur d'améliorer son revenu agricole en parallèle améliorer son niveau de vie. Mais cette bonne intention doit être accompagnée de beaucoup de mesures, notamment la mise en place d'une législation appropriée, et mettre en place des conditions qui permettent aux agriculteurs d'augmenter leur productivité, ce qui réduira le besoin d'une activité secondaire.

Nous avons constaté au cours de notre enquête que la pluriactivité est un phénomène tabou pour la majorité des agriculteurs. La plupart des agriculteurs ont eu peur de s'exprimer sur leur deuxième activité. Selon eux, c'est interdit par la loi. Si on considère que la pluriactivité est déterminante pour la survie des exploitations agricole et des zones rurales, les décideurs politiques doivent combler le vide législatif qui pénalise la pluriactivité. Les agriculteurs doivent non seulement avoir le droit de travailler hors exploitation mais aussi recevoir tous les avantages notamment la sécurité sociale.

Nous avons aussi constaté qu'une partie des agriculteurs enquêtés considèrent le secteur agricole comme un secteur à problèmes et à risques, surtout avec l'absence des équipements. Comme le confirme nos résultats, l'équipement et la productivité réduisent le besoin de travailler hors ferme. Les autorités peuvent donc encourager la modernisation des fermes en Algérie, par exemple en subventionnant et/ou accordant des prêt bancaires pour que les agriculteurs puissent octroyer les équipements nécessaires pour augmenter leur productivité.

Nos résultats empiriques indiquent que l'irrigation est importante. Les décideurs politiques devraient adopter une stratégie pour la gestion des richesses hydraulique naturelles du pays. En particulier, les décideurs responsables du secteur agricoles doivent offrir des aides et des avantages aux petites et moyennes exploitations, pour leur permettre d'éviter la pluriactivité et donc la survie de leurs exploitations.

Une limitation évidente de notre étude est la taille limitée de l'échantillon. Même si nous préconisons la validité de notre échantillonnage, nous reconnaissons l'importance d'un échantillon plus représentatif. En plus des contraintes financières, nous avons eu de sérieuses difficultés à faire en sorte que les répondants révèlent ou non un deuxième emploi. En effet, la plupart des agriculteurs interrogés croyaient que le fait d'avoir un emploi hors ferme était illégal. Il a fallu beaucoup d'effort pour les convaincre à divulguer des informations relatives à la pluriactivité. Ainsi, bien que notre étude soit limitée, notre échantillon est précieux et assez riche pour transmettre des informations pertinentes et fiables sur la pluriactivité.

Notre travail peut être élargi par la considération des liens potentiels entre la pluriactivité et la multifonctionnalité. En effet, l'impact de multifonctionnalité peut être important pour toute décision d'être pluriactif. Par exemple, Rivaroli et al. (2016) propose qu'il est important de tenir compte des caractéristiques internes de l'exploitation. Ces auteurs trouvent que certaines caractéristiques, tel que l'âge et l'éducation sont positivement associés au niveau de multifonctionnalité, et que cette dernière encourage le développement rural. Finalement, il serait aussi intéressant de prendre en considération les caractéristiques de la famille agricole. Galdeano-Gomez et al. (2017) trouve que l'éducation, l'âge, et le revenu sont positivement associé a la multifonctionnalité. Il serait donc intéressant de voir l'impact de ces caractéristiques sur la pluriactivité. 


\section{References}

Abdulai A. and Delgado C.L., 1999. Determinants of Nonfarm Earnings of Farm-Based Husbands and Wives in Northern Ghana. American Journal of Agricultural Economics, 81: 117-130.

Ahituv A. and Kimhi A., 2002. Off-farm work and capital accumulation decisions of farmers over the life-cycle: the role of heterogeneity and state dependence. Journal of Development Economics, 68: 329-353.

Alasia A., Weersink A., Bollman R.D., and Cranfield J., 2009. Off-farm labour decision of Canadian farm operators: Urbanization effects and rural labour market linkages. Journal of Rural Studies, 25: 12-24.

Andersson H., Ramamurtie S., and Ramaswami B., 2003. Labour income and risky investments: can part-time farmers compete? Journal of Economic Behavior \& Organization, 50: 477-493.

Anseeuw W. and Laurent C., 2007. Occupational paths towards commercial agriculture: The key roles of farm pluriactivity and the commons. Journal of Arid Environments, 70: 659-671.

Benjamin C. and Kimhi A., 2006. Farm work, offfarm work, and hired farm labour: estimating a discrete-choice model of French farm couples' labour decisions. European Review of Agricultural Economics, 33: 149-171.

Benjamin C., 1994. The growing importance of diversification activities for French farm households. Journal of Rural Studies, 10: 331-342.

Bojnec S., Dries L., and Swinnen J.F.M., 2003. Human Capital and Labour Flows out of the Agricultural Sector: Evidence from Slovenia. Proceedings of the 25th International Conference of Agricultural Economists (IAAE), 16-22 August 2003. ISBN: 0-958-46098-1 Durban, South Africa.

Bouchakour R. and Bédrani S., 2015. Pluriactivity, the Dutch disease and sustainable agriculture in Algeria. International Journal of Technology Management and Sustainable Development, 14: 241-259.

Brosig S., Glauben T., Herzfeld T. and Wang X ., 2009. Persistence of full- and part-time farming in Southern China. China Economic Review, 20: 360-371.

Carricano M., Poujol F. and Bertrandias L., 2010. Analyse de données avec SPSS. France : Pearson Education.

Cavazzani A. and Fuller A.M., 1982. International perspectives on part-time farming: A review. Geojournal, 6: 383-389.

Chabane M. 2013. Heurs et malheurs du secteur agricole en Algérie 1962-2012, Paris : L'Harmattan.
Chaulet C., 1987. La terre, les frères et l'argent : Stratégie familiale et production agricole en Algérie depuis 1962. Alger (Algerie), OPU. 3, vol. 1198p.

Corsi A. and Salvioni C., 2012. Off- and on-farm labour participation in Italian farm households. Applied Economics, 44: 2517-2526.

Deininger K. and Olinto P., 2001. Rural Nonfarm Employment and Income Diversification in Colombia. World Development, 29: 455-465.

Edmond H. and Crabtree J.R., 1994. Regional variation in Scottish pluriactivity: The Socio-economic context for different types of non-farming activity. Scottish Geographical Magazine, 110: 76-84.

Galdeano-Gomez E., Zepeda-Zepeda J.A., Piedra-Munoz L., Vega-Lopez L.L., 2017. Family farm's features influencing socio-economic sustainability: An analysis of the agri-food sector in southeast Spain. New Medit, 16: 50-61.

Goetz S.J. and Debertin D.L., 2001. Why Farmers Quit: A County-Level Analysis. American Journal of Agricultural Economics, 83: 1010-1023.

Gould B.W. and Saupe W.E., 1989. Off-Farm Labour Market Entry and Exit. American Journal of Agricultural Economics, 71: 960-969.

Gunter L. and McNamara K.T., 1990. The Impact of Local Labour Market Conditions on the Off-Farm Earnings of Farm Operators. Southern Journal of Agricultural Economics, 22: 155-165.

Hearn D.H., McNamara K.T. and Gunther L., 1996. Local economic structure and off-farm labour earnings of farm operators and spouses. Journal of $\mathrm{Ag}$ ricultural Economics, 47: 28-36.

Huffman W.E., 1980. Farm and Off-Farm Work Decision: The Role of Human Capital. The Review of Economics and Statistics, 62: 14-23.

Kimhi A., 2000. Is Part-Time Farming Really a Step in the Way out of Agriculture? American Journal of Agricultural Economics, 82: 38-48.

Lass D.A. and Gempesaw C.M., 1992. The Supply of Off-Farm Labour: A Random Coefficients Approach. American Journal of Agricultural Economics, 74: 400-411.

Liu S., Zhang H., Xie F. and Guo S., 2013. Current Situation and Influencing Factors of Pluriactivity in Mountainous and Hilly Rural Areas of Sichuan Province, China. Journal of Mountain Science, 10: 445-454.

Manceron G. et Aissani F., 1996. Comprendre la Crise. Algérie: Editions Complexe, 232 p.

Markowitz H.M., 1968. Portfolio selection: efficient diversification of investments (Vol. 16). New Haven : Yale University Press. 
Martinez Jr A., Western M., Haynes M., Tomaszewski W. and Macarayan E., 2014. Multiple job holding and income mobility in Indonesia. Research in Social Stratification and Mobility (in press) http://dx. doi.org/10.1016/j.rssm. 2013.09.008.

Matshe I. and Young T., 2004. Off-farm labour allocation decisions in small-scale rural households in Zimbabwe. Agricultural Economics, 30: 175-186.

Ministère de l'Agriculture et de Développement Rural, 2009. Présentation de la politique de Renouveau Agricole et Rural en Algérie et du programme quinquennal 2010-2014. Algérie.

Ministère délégué chargé de développement rural, 2004. Stratégie nationale de développement rural durable. Projet de document, juillet 2004.

Mishra A.K. and Goodwin B.K., 1997. Farm Income Variability and the Supply of Off-Farm Labour. American Journal of Agricultural Economics, 79: 880-887.

Mishra A.K. and Morehart M.J., 2001. Off-farm investment of farm households: A logit analysis. $\mathrm{Ag}$ ricultural Finance Review, 61: 88-101.

Reardon T., Delgado C. and Malton P., 1992. Determinants and Effects of Income Diversification Amongst Farm households in Burkina Faso. Journal of Development Studies, 28: 264-296.

Rivaroli S., Bertazzoli A., Ghelfi R., and Laghi A., 2016. Multifunctional farming in Emilia-Romagna region: an analysis through agricultural census data. New Medit, vol. 15, n. 2: 37-44.
Serra T., Goodwin B.K. and Featherstone A.M., 2005. Agricultural Policy Reform and Off-farm Labour Decision. Journal of Agricultural Economics, 56: 271-285.

Shefrin H. and Statman M., 2000. Behavioral portfolio theory. Journal of Financial and Quantitative Analysis, 35: 127-151.

Simpson W., and Kapitany M., 1983. The Off-Farm Work Behavior of Farm Operators. American Journal of Agricultural Economics, 65: 801-805.

Sofer M., 2001. Pluriactivity in the Moshav: family farming in Israel. Journal of Rural Studies, 17: 363375 .

Sumner D.A., 1982. The Off-Farm Labour Supply of Farmers. American Journal of Agricultural Economics, 64: 499-509.

Tavernier E.M., Temel T.T. and Li F., 1997. The Role of Farm Ownership in Off-Farm Work Participation. Agricultural and Resource Economics Review, 26: 67-81.

Woldehanna T., Oude-Lansink A. and Peerlings J., 2000. Off-farm work decisions on Dutch cash crop farms and the 1992 and Agenda 2000 CAP reforms. Agricultural Economics, 22: 163-171.

World Bank, 1997. Rural Development: From Vision to Action. Environmentally and Socially Sustainable Development Studies and Monographs Series 12. Washington, DC: The World Bank.

Zhao J., 2014. Rural income diversification patterns and their determinants in China. Agricultural Economics - Czech, 60: 219-231. 